\title{
Implicit Flux-Split Schemes for the Euler Equations
}

\author{
James L. Thomas* \\ NASA Langley Research Center, Hampton, Virginia \\ Bram van Leer $\dagger$ \\ University of Michigan, Ann Arbor, Michigan \\ and \\ Robert W. Waltersł \\ Virginia Polytechnic Institute and State University, Blacksburg, Virginia
}

\begin{abstract}
$\mathbf{P}$ ROGRESS in the development of implicit algorithms for the Euler equations using the flux vector splitting method is described. Comparisons of the relative efficiency of relaxation and spatially split, approximately factored methods on a vector processor for transonic and supersonic twodimensional channel flows are made. A hybrid threedimensional algorithm is developed that uses relaxation in one coordinate direction and approximate factorization in the crossflow plane. The scheme is completely vectorizable and recovers conventional space-marching schemes for fully supersonic flows. The method is applied to a forebody shape in supersonic flow with an embedded pocket of subsonic flow.
\end{abstract}

\section{Contents}

\section{Two-Dimensional Algorithms}

Upwind-differencing methods have been successfully used to obtain solutions to the Euler equations for flows with strong shocks. These methods approximate the signalpropagation features of hyperbolic equations and are naturally dissipative, so that no artificial viscosity terms are required. More computational work per time step is required than with central-difference methods, which can be offset by the possibilities for increased versatility and improved convergence rates per time step.

The upwind-differencing scheme considered is based on the concept of flux vector splitting and uses the splitting of Van Leer. A general class of semidiscrete spatial-differencing schemes is considered, ranging from first- or second-order fully upwind schemes to third-order upwind-biased differencing. The two basic implicit schemes considered in two dimensions are both approximations to the unfactored backward-time integration scheme in delta form and correspond to a spatially split approximate-factorization (AF) method ${ }^{\prime}$ and the classical Gauss-Seidel relaxation method, ${ }^{2,3}$ respectively.

The AF method has the advantage in that it is a general, time-accurate integration scheme, applicable to a wide class of problems. The scheme is practically independent of the type of spatial differencing and is vectorizable on current pipeline

Received Feb. 3, 1986; revision received Sept. 15, 1988. Copyright (C) 1988 American Institute of Aeronautics and Astronautics, Inc. No copyright is asserted in the United States under Title 17, U.S. Code. The U.S. Government has a royalty-free license to exercise all rights under the copyright claimed herein for Governmental purposes. All other rights are reserved by the copyright owner. Full paper available from National Technical Information Service, Springfield, VA 22151, at standard price (available upon request).

*Senior Research Scientist, Analytical Methods Branch, Low-Speed Aerodynamics Division. Associate Fellow AIAA.

†Professor, Department of Aerospace Engineering. Member AIAA.

łAssistant Professor, Department of Aerospace and Ocean Engineering. Member AIAA. computers. The disadvantage of the scheme is that the allowable time step is limited. In two dimensions, the scheme is unconditionally stable, but the optimum convergence rate for steady-state calculations is generally obtained at a value of the time step on the order of a Courant number of 10 , although the optimal value is problem dependent. In three dimensions, the spatially factored algorithm is only conditionally stable for upwind differencing; the maximum allowable time step is on the order of the value used in an explicit scheme.

The relaxation algorithms considered are tailored to the form of the coefficient matrix arising in the unfactored implicit scheme when using upwind differencing. The present study has concentrated on classical line Gauss-Seidel relaxation, with the provision that alternating sweep directions are used to maintain stability in subsonic flow with higher-order differencing. The scheme is unconditionally stable in a scalar linear stability analysis, both in two and three dimensions. For supersonic flow in two dimensions, with fully upwind differencing, the algorithm recovers Newton's method for large time steps. The disadvantage of the relaxation schemes is that they are not completely vectorizable on vector processors.

The relative efficiencies of the two approaches have been compared on the basis of a number of test cases. For a transonic $(M=0.85)$ channel flow, the higher convergence rate per iteration of the relaxation algorithms is amply compensated for by the faster computational rate per iteration of the $\mathrm{AF}$ algorithm. For a mesh of $85 \times 41$ points, the optimal time step for the AF algorithm is a Courant number of 10; for the relaxation scheme, the optimal time step is on the order of 100 and $10^{5}$ for the vertical and horizontal line algorithms, respectively. The time step limitation encountered for the vertical line relaxation scheme is very sensitive to the implementation of boundary conditions and worsens as the grid is refined. Using a Fourier analysis of a coupled two-dimensional linear system as a model for the Euler equations, a recent study by Mulder $^{3}$ confirms that the line Gauss-Seidel scheme is not unconditionally stable. On sufficiently fine grids, underrelaxation is required for stability, in accordance with the present numerical results.

For a supersonic $(M=1.65)$ flow in a channel, the linerelaxation algorithm with fully upwind differencing reduced the residual 12 orders of magnitude in 25 iterations, compared to 200 iterations for the AF scheme. The relaxation method is more efficient as the Mach number is increased since the numerical domain of dependence is more closely matched to the physical domain of dependence; efficient strategies to handle local regions of embedded subsonic flow are also possible with the relaxation scheme.

\section{Three-Dimensional Algorithm}

A three-dimensional algorithm based on either approximate factorization or line relaxation is possible. With upwind differencing of the residual, the spatially split AF algorithm can be shown to be only conditionally stable for the scalar convec- 
tion equation but has the advantage of being completely vectorizable. The line-relaxation algorithms can be shown to be unconditionally stable for the scalar convection equation but are not completely vectorizable. A hybrid approach is adopted here, using relaxation in one coordinate direction and approximate factorization in the remaining crossflow plane. The hybrid approach avoids the $(\Delta t)^{3}$ splitting error in the threedimensional AF approach, which is responsible for the severe time step limitation. The hybrid algorithm is completely vectorizable and can recover conventional space-marching techniques for fully supersonic flow.

The linearized, backward-time approximation in delta form for the three-dimensional equation in generalized coordinates

$$
\left[\frac{I}{J \Delta t}+\delta_{\xi} \frac{\partial \hat{F}}{\partial Q}+\delta_{\eta} \frac{\partial \hat{G}}{\partial Q}+\delta_{\zeta} \frac{\partial \hat{H}}{\partial Q}\left[\Delta Q=R^{n}\right.\right.
$$

represents a large banded $5 \times 5$ block matrix equation. Here, $Q$ is the vector of conserved variables, $\hat{F}, \hat{G}$, and $\hat{H}$ the generalized fluxes, and $J$ the Jacobian of the transformation. Applying upwind relaxation in the $\xi$ direction leaves the following equation to be solved in the crossflow $(\eta-\zeta)$ plane:

$$
\left[M+\delta_{\eta} \frac{\partial \hat{G}}{\partial Q}+\delta_{\zeta} \frac{\partial \hat{H}}{\partial Q}\right] \Delta Q=R\left(Q^{n}, Q^{n+1}\right)
$$

where

$$
M=\frac{I}{J \Delta t}+\frac{\partial \hat{F}^{+}}{\partial Q}-\frac{\partial \hat{F}^{-}}{\partial Q}
$$

includes the time term and the diagonal blocks from implicit discretization in the $\xi$ direction, here taken as first-order accurate differencing. Nonlinear updating of the residual $R$ is indicated, corresponding to the use of updated upstream crossflow planes when sweeping in the downstream direction and vice versa. The streamwise relaxation is effected by sweeping the $\xi$ direction through the mesh, alternating between forward and backward passes in order to maintain stability for higher-order differencing.

The crossflow plane equation is solved with a spatially split AF algorithm,

$$
\begin{aligned}
& {\left[M+\delta_{\eta} \frac{\partial \hat{G}}{\partial Q}\right] M^{-1}\left[M+\delta_{\zeta} \frac{\partial \hat{H}}{\partial Q}\right] \Delta Q^{n}} \\
& =R\left(Q^{n+1}, Q^{n}\right)
\end{aligned}
$$

From a stability consideration, there is no particular advantage in using upwind discretization in the crossflow plane, as long as upwind differencing is used in the $\xi$ direction. In particular, one might use central differencing in the crossflow plane, although the present results were all obtained with the flux splitting approach.

For fully supersonic flows, the upstream sweep can be eliminated, in alalogy with the physical domain of dependence. The extension to thin-layer and supersonic parabolized viscous flows is straightforward, as the implicit terms in either of the $\eta$ or $\zeta$ directions are solved simultaneously. Closely related is the two-factor scheme resulting from an eigenvalue factorization in the $\xi$ direction, which has the advantage of being a single step time-accurate scheme.

\section{Results}

Supersonic flow at Mach 1.7 over an analytically defined forebody, contoured to be representative of the cockpit region of a high-performance fighter, is considered; pressure data at a nominal Reynolds number of $3 \times 10^{6}$ based on body length are available. ${ }^{4}$ The grid consisted of 31 points in the axial direction, 38 points circumferentially, and 31 points normal to

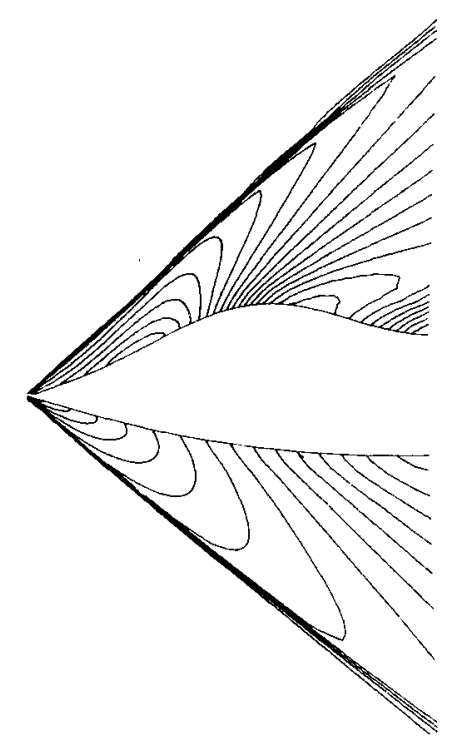

Fig. 1 Mach contours for analytic forebody $(M=1.7, \alpha=0 \mathrm{deg})$.

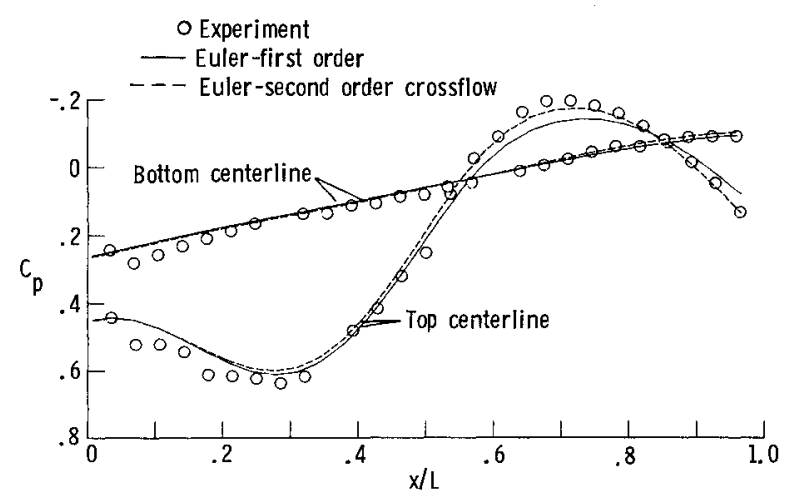

Fig. 2 Pressure distributions for analytic foregody $(M=1.7, \alpha=-5$ deg).

the surface. The Mach contours along the longitudinal plane of symmetry are shown in Fig. 1 at 0 deg angle of attack. The flow is fully supersonic in the streamwise direction and can be efficiently computed by marching in space.

At sufficiently low negative incidence, however, there are regions in which the Mach number in the marching direction becomes subsonic and space marching is no longer possible. Computed results for such a situation at $-5 \mathrm{deg}$ angle of attack are shown in Fig. 2. The pressure distribution is accurately predicted with the second-order accurate discretization in the crossflow plane.

The algorithm has been extended to compute transonic flows over a swept wing at the angle of attack by applying the relaxation in the spanwise coordinate direction. Also, conical flow solutions can be recovered with the present method and a series of delta wings have been computed.

\section{References}

${ }^{1}$ Beam, R. M. and Warming, R. F., "An Implicit Factored Scheme for the Compressible Navier-Stokes Equations," AIAA Journal, Vol. 16, April 1978, pp. 393-402.

${ }^{2}$ Chakravarthy, S. R., "Relaxation Methods for Unfactored Implicit Upwind Schemes," AIAA Paper 84-0165, Jan. 1984.

${ }^{3}$ Mulder, W. A., "A Note on the Use of Symmetric Line GaussSeidel for the Steady Upwind Differenced Euler Equations," Stanford Univ., Stanford, CA, Rept. Classic 87-20, 1987.

${ }^{4}$ Townsend, J. C., Howell, D. T., Collins, I. K., and Hayes, C., "Surface Pressure Data on a Series of Analytic Forebodies at Mach Numbers from 1.70 to 4.50 and Combined Angles of Attack and Sideslip," NASA TM 80062, June 1978. 